\title{
THE ASSOCIATION OF IRIDOSCHISIS AND PRIMARY ANGLE-CLOSURE GLAUCOMA
}

\author{
J. F. SALMON AND A. D. N. MURRAY \\ Cape Town, South Africa
}

\begin{abstract}
SUMMARY
Twelve patients with iridoschisis in one or both eyes were studied to determine the clinical features of the condition and to examine the relationship of iridoschisis to primary angle-closure glaucoma. A spectrum of iris pathology, from subtle intrastromal atrophy to extensive splitting of the anterior layer of iris with fibrillar disintegration, was found in the affected and fellow eyes. Gonioscopy revealed partial or complete angle closure, particularly involving the superior angle, in all patients. Seven had glaucomatous disc damage and five had normal discs. The mean axial length and anterior chamber depth measurements in patients with iridoschisis were similar to those found in matched patients with primary angleclosure glaucoma but were significantly less than the measurements found in matched normals $(p<0.001)$. This study suggests that iridoschisis is an unusual manifestation of iris stromal atrophy and results from intermittent or acute elevation of intraocular pressure. Primary angle-closure glaucoma should be excluded in patients who present with iridoschisis.
\end{abstract}

In 1945, Loewenstein and Foster proposed the term "iridoschisis" to describe a localised cleavage of the iris stroma into two layers, which had occurred in an elderly patient with absolute glaucoma. ${ }^{1}$ The anterior leaf of iris was atrophic and had disintegrated into fibrils which were floating freely in the anterior chamber.

Since the original study by Schmitt in 1922, most descriptions of iridoschisis are limited to individual case reports. ${ }^{1-15}$ However, two series have been reported, each describing six patients. ${ }^{16-17}$ Although the pathogenesis of iridoschisis is unknown, it has been associated mainly with angle-closure glaucoma or has occurred as an apparently idiopathic atrophy in aged eyes. Other less common associations include congenital abnormalities, congenital syphilis or a history of antecedent trauma. ${ }^{12,13,18,19}$

From: the Department of Ophthalmology, University of Cape Town \& Groote Schuur Hospital, Cape Town, South Africa.

Correspondence to: Dr. J. F. Salmon FRCS, FCOphth, Department of Ophthalmology, Medical School, University of Cape Town, Observatory 7925, Cape Town, South Africa.
In this report, we describe the clinical features found in twelve patients with iridoschisis and discuss the relationship of iridoschisis to raised intraocular pressure in primary angleclosure glaucoma.

\section{PATIENTS AND METHODS}

During a five-year period, from June 1985 to May 1990, a total of seventeen patients with iridoschisis were seen at Groote Schuur Hospital, Cape Town. Twelve of these patients were referred to the glaucoma clinic because of acutely raised intraocular pressure or because of glaucomatous disc damage with visual field loss and two were referred because of unusual iris changes. Three patients were seen once in a general ophthalmic clinic and failed to return for follow-up. Because of insufficient clinical details on retrospective analysis these three patients have been excluded from this report. (Subsequently it was learnt that one had died and the other two could not be traced).

We therefore examined fourteen unrelated patients with iridoschisis in one or both eyes and were responsible for their treatment. Two patients with a history of ocular trauma and angle recession glaucoma will be reported separately. Of the remaining twelve patients, three were males and nine females. Their ages ranged from 39 to 76 years (mean 63.3 years).

A detailed ophthalmic and medical history was taken. Each patient underwent a full ophthalmic examination, including best corrected visual acuity, slit lamp examination, applanation tonometry, gonioscopy and direct ophthalmoscopy. Humphrey computerised field analysis was performed and A-scan ultrasound was used to measure the corneal thickness-anterior chamber depth and the axial length. Anterior segment photography was performed in all patients.

To describe the anterior chamber angle, both Scheie's gonioscopic classification (based on the extent of visible angle structures) and Shaffer's classification (based on the degrees of arc subtended by the inner surface of the trabec- 
ular meshwork and the anterior surface of the iris) were used. ${ }^{20}$ By using both classifications, an accurate description could be achieved for those patients with a flatter iris who nevertheless had closed angles. In Scheie's classification, narrow angles are graded between I and IV. Grade I implies a difficulty to see over the iris root into the recess; Grade II implies an obscured ciliary body band; Grade III implies an obscured posterior trabeculum and Grade IV (closed) implies that only Schwalbe's line is visible. In Schaffer's classification, Grade 1 represents a geometric angle of $10^{\circ}$, Grade 2 an angle of $20^{\circ}$, Grade 3 an angle of $25-35^{\circ}$ and Grade 4 an angle of $35-45^{\circ}$.

Ocular biometry results for the study patients were compared with two control groups who were matched for age, sex and race. The one control group consisted of 24 patients of mixed ethnicity, with a mean age of 63.7 years, who had primary angle-closure glaucoma without iris pathology. The other control group consisted of 24 healthy individuals of mixed ethnicity, with a mean age of 60.3 years who had no evidence of ocular pathology or refractive error. In patients with iridoschisis, axial length measurements were obtained in 19 eyes and anterior chamber depth measurements in 17 eyes. The axial length and anterior chamber depth were measured in both eyes of all patients in the control groups. Statistical analysis of variance was used to compare the measurements. A finding was considered significant at $\mathrm{P}<0.05$.

\section{RESULTS}

Of the twelve patients in this study, six presented initially with a history of gradual, painless loss of vision or with presbyopic symptoms. Six presented with painful loss of vision, including one who had undergone an enucleation in 1979 for absolute glaucoma resulting from untreated acute angle-closure.

Ten patients (patients 1-10) had suffered from intermittent headaches or eye pain, for periods ranging from a few months to sixteen years. On presentation, the intraocular pressure in these patients ranged from $12 \mathrm{mmHg}$ to $65 \mathrm{mmHg}$ (mean $35 \mathrm{mmHg}$ ). In fourteen eyes (seven patients) there was evidence of glaucomatous disc damage with a corresponding field defect, but in five eyes (three patients) no damage to the optic nerve was detected. All ten patients had gonioscopic evidence of angle closure, particularly involving the superior angle and all experienced symptomatic relief with control of the intraocular pressure (Table I).

In two patients, no previous headaches or eye pain had been experienced. One of these patients presented with acute glaucoma after pupillary dilation (patient 11 ). One presented with visual loss secondary to a cataract and was coincidentally noted to have inferior iridoschisis, a closed superior angle but a normal optic disc (patient 12).

Eleven patients had heavily pigmented, brown irides and one had brown-grey irides. Seven had iridoschisis in both eyes and five in one eye. The inferior quadrants were involved in 17 eyes (Figs 1 and 2) and both the superior and inferior quadrants in two eyes (Figure 3 ). Iris debris was seen in the inferior angles of three eyes (patients 4 and 10 bilaterally).

The area of iridoschisis extended to the pupil margin in six eyes. A fixed dilated pupil was present in one eye, but the remainder had normal, round pupils. The loose strands of iris tissue resulted in limited corneal touch in 12 eyes, with a localised corneal opacity in one and a decompensated, oedematous cornea in another. One patient had signs of interstitial keratitis. Clear lenses were present in only two eyes (patients 5 and 10) and one lens was subluxed (patient 10). Nuclear sclerosis or corticular lens opacities were present in all the other eyes. Some had fine anterior subcapsular lens opacities suggestive of previous glaukomflecken.

In the fellow eyes of four patients with unilaterial iridoschisis, subtle atrophic changes were noted in the inferior stroma of the iris (Table I). Superficial intrastromal cystic changes were present and in addition, in two patients, fine slits were observed in the anterior surface of the iris overlying the cystic changes (Figure 1). Two of these four eyes (in patients 3 and 12) had normal discs and two (in patients 2 and 7) had cupped discs.

The A-scan ultrasound measurements obtained from patients with iridoschisis were compared with the measurements obtained in matched patients with primary angle-closure glaucoma and matched normal individuals. The anterior chamber depths were measured from the anterior surface of the cornea to the anterior surface of the lens (Fig. 4). The mean axial length of the eyes of patients with iridoschisis was $22.15 \mathrm{~mm}$ (standard deviation 0.70) and the mean anterior chamber depth was $2.52 \mathrm{~mm}$ (standard deviation 0.40 ). In matched patients with primary angle-closure glaucoma without iris changes, the mean axial length of 48 eyes was $22.42 \mathrm{~mm}$ (standard deviation 0.61 ) and mean anterior chamber depth was $2.45 \mathrm{~mm}$ (standard deviation 0.25 ). The measurements obtained in these two groups were statistically similar. In matched normal patients, the mean axial length was $23.14 \mathrm{~mm}$ (standard deviation 0.55) and mean anterior chamber depth was $2.81 \mathrm{~mm}$ (standard deviation 0.30 ). There was a highly significant difference between the measurements found in normal individuals and those found in patients with iridoschisis or primary angle-closure glaucoma $(\mathrm{p}<0.001)$.

The intraocular pressure was controlled medically in all patients before surgery was performed. Eight patients (fourteen eyes) underwent a YAG laser peripheral iridotomy. In three patients a trabeculectomy was performed and one subsequently underwent uniocular implantation of a Molteno tube because of persistently raised intraocular pressure. Two patients had a cataract extraction with intraocular lens implantation and iridectomy as primary surgical treatment (patients 3 and 12). In one, a corneal graft was performed at the same time as the cataract extraction. Cataract surgery was subsequently performed in a further five patients during the five-year period of follow-up (Table II). After surgery, the structures of the superior angle were clearly visible in all except two 
Table I. Clinical data for fourteen patients with iridoschisis

\begin{tabular}{|c|c|c|c|c|c|c|c|c|c|c|c|}
\hline \multirow[b]{2}{*}{ Patient } & \multirow[b]{2}{*}{ Race } & \multirow[b]{2}{*}{ Age } & \multirow[b]{2}{*}{ Sex } & \multicolumn{2}{|c|}{ I.O.P. $[\mathrm{mmHg}]^{*}$} & \multicolumn{2}{|c|}{ Cup: Disc Ratio } & \multicolumn{2}{|c|}{ Iridoschisis§ } & \multicolumn{2}{|c|}{ Angle $†$} \\
\hline & & & & $\mathrm{R}$ & $\mathrm{L}$ & $\mathrm{R}$ & $\mathrm{L}$ & $\mathbf{R}$ & $\mathrm{L}$ & $\mathrm{R}$ & $\mathrm{L}$ \\
\hline 1 & $\mathbf{M}$ & 57 & M & 65 & 50 & 1.0 & 0.5 & SUP/INF & INF & IV $180^{\circ}(1)$ & IV $180^{\circ}(1)$ \\
\hline 3 & M & 60 & $\mathrm{~F}$ & 12 & 14 & 0.1 & 0.1 & NIL & INF & IV $180^{\circ}(1)$ & IV $90^{\circ}(1)$ \\
\hline 4 & $\mathrm{M}$ & 75 & $\mathrm{~F}$ & 40 & 48 & 0.9 & 0.7 & INF & INF & IV $270^{\circ}(2)$ & IV $270^{\circ}(2)$ \\
\hline 5 & W & 69 & $\mathrm{~F}$ & 21 & 21 & 0.5 & 0.6 & INF & INF & IV $180^{\circ}(1)$ & IV $180^{\circ}(1)$ \\
\hline 6 & M & 54 & M & - & 40 & - & 0.4 & - & INF & - & IV $180^{\circ}(2)$ \\
\hline 7 & $\mathbf{M}$ & 68 & $\mathrm{~F}$ & 16 & 19 & 0.7 & 0.8 & NIL & INF & IV $180^{\circ}(1)$ & IV $90^{\circ}(1)$ \\
\hline 8 & $\mathbf{M}$ & 49 & $\mathrm{~F}$ & 43 & 32 & 0.1 & 0.1 & INF & INF & IV $360^{\circ}(1)$ & IV $360^{\circ}(1)$ \\
\hline 9 & $\mathrm{M}$ & 68 & $\mathrm{~F}$ & 40 & 55 & 0.8 & 0.9 & INF & INF & IV $360^{\circ}(1)$ & IV $360^{\circ}(1)$ \\
\hline 10 & $\mathbf{M}$ & 39 & $\mathrm{~F}$ & 55 & 40 & 0.8 & 1.0 & SUP/NIL & INF & IV $180^{\circ}(1)$ & IV $180^{\circ}(1)$ \\
\hline 11 & $\mathbf{M}$ & 76 & $\mathrm{~F}$ & 12 & 60 & 0.3 & 0.5 & INF & INF & III $180^{\circ}(1)$ & IV $180^{\circ}(1)$ \\
\hline 12 & $\mathbf{M}$ & 72 & M & 9 & 10 & 0.1 & 0.1 & NIL & INF & III $360^{\circ}(2)$ & IV $180^{\circ}(2)$ \\
\hline
\end{tabular}

Age in years. Race: $M$ indicates mixed racial ancestry, $W$ indicates white. Sex: $M$ indicates male, $F$ indicates female.

*Intraocular pressure on presentation.

§SUP indicates superior iris involvement. INF indicates inferior iris involvement. NIL indicates iris atrophy without iridoschisis.

$\uparrow$ Angle description based on Scheie's classification: III indicates posterior trabeculum obscured. IV indicates only Schwalbes' line visible.

The maximum amount of angle closure on presentation is indicated in degrees. Shaffers' classification in brackets.

patients (patients 1 and 6 ) who had evidence of peripheral anterior synechiae. Two patients (patients 9 and 10) did not return for follow up after their initial period of therapy. In the remainder, progressive iris change was not noticed. Long term intraocular pressure control was obtained in all patients, although topical pilocarpine $1 \%$ was required after peripheral iridotomy in four patients.

Nine of the twelve patients had systemic hypertension and in two the diagnosis of congenital syphilis was made (patients 6 and 7). One other patient had a positive serological test for syphilis (patient 12).

\section{DISCUSSION}

During a five-year period, an attempt was made to examine all the patients with iridoschisis who had presented to this hospital. This series describes twelve patients with primary angle-closure glaucoma associated with iridoschisis in one or both eyes. In all involved eyes, the anterior leaf of iris had split from the underlying iris stroma and had disintegrated into fibrils which projected into the anterior chamber. Although all the patients had involvement of the inferior quadrants of the iris, two also had involvement of the superior quadrants. These characteristic signs of iridoschisis have been described and illus-

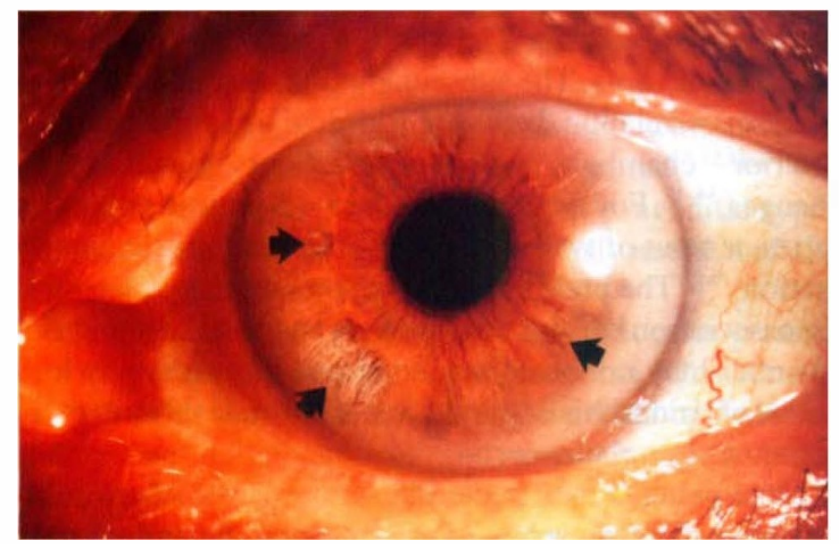

Fig. 1. Patient 12. Left eye demonstrating the earliest form of iris atrophy and iridoschisis (arrows). trated in the past, either in association with angle-closure glaucoma or as an apparently idiopathic atrophy of old age. ${ }^{1-12,14-17}$

The pupil was round and central in all except one eye, where a fixed and dilated pupil was found. Because of iris touch, one patient had developed a localised corneal opacity and another an oedematous cornea; a complication that has been previously noted in patients with iridoschisis. ${ }^{15,17}$ In four patients, subtle atrophy was present in the inferior iris stroma of the fellow eye suggestive of early, incomplete iridoschisis.

The diagnosis of primary angle-closure glaucoma was based on gonioscopic evidence of angle closure, particularly involving the superior angle; a shallow anterior chamber and a short axial length. Nuclear sclerosis influenced the refraction found on presentation in some patients, who were less hypermetropic than their axial length measurements would suggest. The mean anterior chamber depth and mean axial length measurements obtained from patients with iridoschisis are similar to previously reported biometric parameters demonstrated in patients with angle-closure glaucoma. ${ }^{21}$ These measurements were similar to those found in a matched group of patients with primary angle-closure glaucoma and statis-

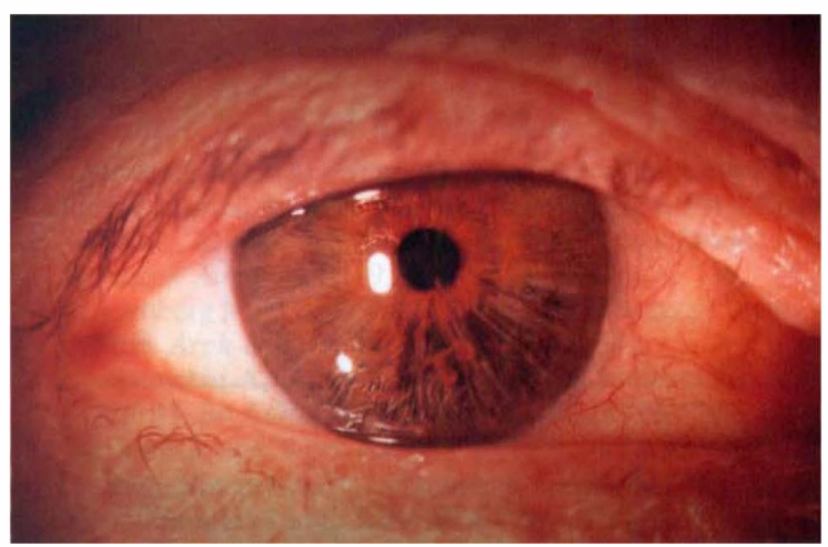

Fig. 2. Patient 5. Right eye demonstrating typical inferior iridoschisis. This appearance was present in most eyes. 


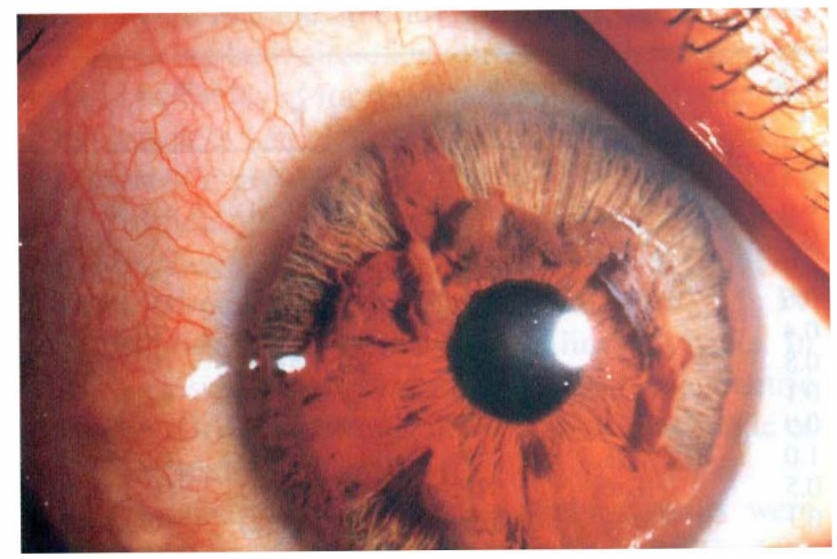

Fig. 3. Patient 10. Right eye demonstrating an advanced form of iridoschisis.

tically significantly different to the findings in matched normals.

Although six patients were asymptomatic on presentation, the history in five was suggestive of subacute angleclosure. Similarly, five of the patients who presented with signs of acute glaucoma gave a history of previous intermittent pain and headache. Examination revealed glauco-
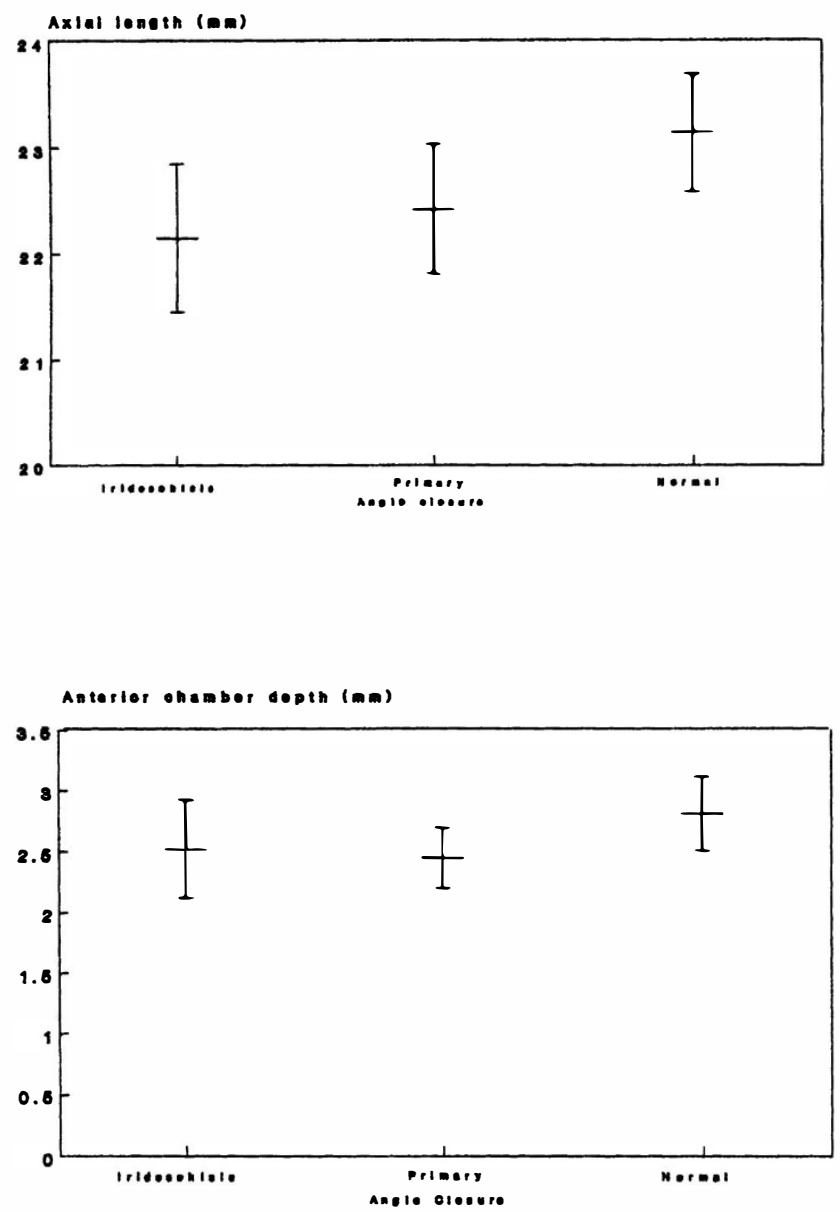

Fig. 4. A-scan ultrasound results of patients with iridoschisis, primary angle-closure glaucoma and matched normals; showing mean values with one standard deviation. The anterior chamber depths are measured from the anterior surface of the cornea to the anterior surface of the lens. matous disc damage and visual field loss in fourteen eyes, but no disc damage was found in nine eyes.

Considering the underlying diagnosis of these patients, it seems likely that raised intraocular pressure may have been responsible in some way for the development of the iridoschisis. Several studies support this suggestion. Anderson and Davis demonstrated that the first permanent effect of acutely raised intraocular pressure in laboratory animals was avascular necrosis of the iris stroma ${ }^{22}$ Histological examination of specimens taken from patients with iridoschisis has demonstrated marked iris stromal atrophy without evidence of vascular or neural alterations. ${ }^{1,17}$ Similarly, fluorescein angiography of the iris has revealed normal vessel perfusion, with normal iris vessels. ${ }^{23}$

Winstanley, in a detailed description of iris atrophy in primary glaucoma, described "iridoschisis-like" changes which had occurred secondary to raised intraocular pressure. ${ }^{24}$ The different iris changes found in the involved and fellow eyes of our patients, suggests that a range of atrophic iris stromal changes may occur secondary to high intraocular pressure. Despite the remarkable appearance of iridoschisis, these characteristic changes may simply be a late and unusual manifestation of iris stromal atrophy.

The question of whether iridoschisis in angle-closure glaucoma is secondary to raised intraocular pressure or is a coincidental finding, is controversial. The patients studied by Posner and by Mills developed iridoschisis after an episode of raised intraocular pressure. ${ }^{9.12}$ On the other hand, after examination of six cases of iridoschisis with associated angle-closure glaucoma, Romano et al. came to the conclusion that the iridoschisis was unlikely to be a sequel of the glaucoma, because the iris changes preceded the acute angle-closure attack. ${ }^{16}$ Considering the fact that ten of our patients $(83 \%)$ had episodes of subacute angleclosure, the presence of iris changes before a typical episode of acute angle-closure glaucoma may be explained by prior episodes of asymptomatic intermittent intraocular pressure elevation.

Mills, in his analysis of the first seventeen reported cases of iridoschisis, found that nine had features consistent with the diagnosis of angle-closure glaucoma and that in seven cases the nature of the glaucoma was not clear. ${ }^{12} \mathrm{He}$ suggested that the relationship between glaucoma and iridoschisis might be specific to primary angleclosure glaucoma. The subsequent literature demonstrates a close correlation between iridoschisis and a shallow anterior chamber, with or without glaucomatous changes. ${ }^{14-17}$ For example, in the two largest published series, ten out of twelve cases had this anatomical configuration. ${ }^{16,17}$ The present study supports the suggestion that the association between iridoschisis and a shallow anterior chamber may not be coincidental and that most previous cases of iridoschisis may have occurred secondary to angle-closure glaucoma.

Most patients with acute glaucoma do not develop iridoschisis. The presence of iris atrophy depends on the duration and severity of the intraocular pressure rise, as well as upon the type of iris pigmentation. ${ }^{24}$. Many of our 
Table II. Refraction and ocular surgery

\begin{tabular}{|c|c|c|c|c|c|c|c|c|}
\hline \multirow[b]{2}{*}{ Patient } & \multicolumn{2}{|c|}{ Visual acuity* } & \multicolumn{2}{|c|}{ Refraction $\dagger$} & \multirow[t]{2}{*}{ Iridotomy } & \multirow[t]{2}{*}{ Trabeculectomy } & \multirow{2}{*}{$\begin{array}{l}\text { Cataract } \\
\text { Extraction }\end{array}$} & \multirow[b]{2}{*}{ Other } \\
\hline & $\mathrm{R}$ & $\mathrm{L}$ & $\mathrm{R}$ & L & & & & \\
\hline 1 & NPL & $6 / 18$ & - & +1.50 & & Left & Left & Left Molteno implant \\
\hline 2 & $6 / 24$ & $6 / 24$ & +2.50 & +1.75 & Right/Left & & Left & \\
\hline 3 & CF & $\mathrm{CF}$ & - & - & & & Right/Left & Left corneal graft \\
\hline 4 & $\mathrm{CF}$ & $6 / 9$ & -0.50 & -0.25 & Right/Left & & Right & \\
\hline 5 & $6 / 9$ & $6 / 12$ & +0.75 & -0.25 & Right/Left & & & \\
\hline 6 & - & $6 / 24$ & - & +2.75 & Left & & & Right enucleation \\
\hline 7 & $6 / 24$ & $\mathrm{CF}$ & +0.75 & - & Right/Left & & & \\
\hline 8 & $\mathrm{CF}$ & $6 / 18$ & +1.00 & +1.00 & Right/Left & & Right/Left & \\
\hline 9 & $6 / 18$ & NPL & +1.00 & - & & Right & & \\
\hline 10 & $6 / 9$ & NPL & - & - & Left & Right & & \\
\hline 11 & $6 / 18$ & $6 / 12$ & +1.00 & +1.25 & Right/Left & & Right/Left & \\
\hline 12 & $6 / 12$ & $\mathrm{CF}$ & +1.00 & +1.00 & & & Right/Left & \\
\hline
\end{tabular}

*NPL indicates no perception of light, CF indicates counting fingers.

$\dagger$ Equivalent sphere. No refraction obtained in blind eyes or eyes with cataract.

patients had heavily pigmented irides and presented with advanced glaucoma; a feature noted particularly in Asia in patients with subacute angle-closure glaucoma. ${ }^{21}$ Iris sphincter damage with tangential distortion of the stroma did not occur in these patients and most had normal pupillary function. This finding has been noted before in eyes with iridoschisis and caused Alberts and Klien to speculate that normal pupillary movements in the presence of iris stromal atrophy might play a role in the development of iridoschisis. ${ }^{10}$

It has been proposed that iris pigment and degenerative material may occlude the trabecular meshwork in some eyes with iridoschisis and reduce the outflow of aqueous from an already compromised drainage system. ${ }^{17}$ The post-operative intraocular pressure control in our patients suggests that this mechanism may not have played a significant role in producing their glaucoma. Any tendency for debris to occlude the trabecular meshwork would have resulted in an intermittent or constant post-operative intraocular pressure elevation in the seven patients (thirteen eyes) who had a peripheral iridotomy only, and in the two patients (four eyes) who underwent an extracapsular cataract extraction with intraocular lens as primary treatment. However, the intraocular pressure was easily controlled for a mean period of three years in ten eyes without additional glaucoma medication and in seven eyes with pilocarpine $1 \%$ drops alone. No further disc damage occurred during the period of follow-up.

Iridoschisis has been previously described in association with other conditions. ${ }^{12,13,18,19}$ Single cases of iridoschisis secondary to trauma, with and without intraocular pressure elevation have been reported..$^{3,12,13,23}$ Two patients in this series had suffered from congenital syphilis, an association that has been previously noted, and nine patients had systemic hypertension. ${ }^{19}$

Our primary aim was to control the intraocular pressure and to prevent progression of the glaucoma. This aim was largely achieved by means of surgery, although medical therapy was initially utilised, and in some cases continued. We found it unnecessary to treat the iridoschisis per se; an approach advocated by Duke-Elder. ${ }^{25}$ Progressive iris changes were not seen and the cornea remained clear in the ten patients who were regular clinic attenders.
The differential diagnosis of iridoschisis includes the iridocorneal endothelial (ICE) syndrome and the Axenfeld-Rieger syndrome. ${ }^{26-28}$ In most cases of the ICE syndrome, the age of onset is earlier, the condition is unilateral, an ectopic pupil with peripheral anterior synechiae is found and iris stromal atrophy with hole formation may occur. Axenfeld-Rieger syndrome is a congenital abnormality occurring in both eyes. Schwalbe's line is prominent, mild iris stromal thinning occurs which may progress to atrophy and hole formation, and corectopia with ectropion uvea may be observed. These conditions were specifically excluded in our patients.

We suggest that "iridoschisis" is simply an unusual manifestation of atrophy of the iris, and in most cases is the result of high intraocular pressure. A spectrum of iris changes may be found, from intrastromal atrophy to extensive splitting of the anterior layer of the iris with disintegration into fibrils. While iris pigment and material may occlude the trabecular meshwork in some eyes, this mechanism probably does not play a significant role in producing glaucoma. Primary angle-closure glaucoma should specifically be excluded in patients who present with iridoschisis.

This study was supported by grants from the Medical Research Council of South Africa and the University of Cape Town.

Key words: Angle-closure glaucoma, axial hypermetropia, intraocular pressure, iridoschisis, iris atrophy.

\section{REFERENCES}

1. Loewenstein A and Foster J: Iridoschisis with multiple rupture of stromal threads. Br J Ophthalmol 1945, 29: 277-82.

2. Schmitt A: Ablösung des vorderen Irisblattes. Klin Monatsbl Augenheilk 1922, 68: 214-5.

3. Schoenberg MJ: Case of detachment of the anterior layers of the iris. Arch Ophthalmol 1927, 56: 538-42.

4. Loewenstein A, Foster J, Sledge SK: A further case of iridoschisis. Br J Ophthalmol 1948, 32: 129-34.

5. Garden RR and Wear AR: Iridoschisis in a case of chronic primary glaucoma. Br J Ophthalmol1949, 33: 509-11.

6. McCulloch C: Iridoschisis as a cause of glaucoma. Am J Ophthalmol 1950; 33: 1398-400.

7. Háik GM, Lyda W, Waugh RL: Iridoschisis. Report of an additional case. Arch Ophthalmol 1952, 48: 40-3. 
8. Carter LF: Progressive senile fibrillar atrophy of the iris stroma (iridoschisis). Am J Ophthalmol 1953, 36: 967-70.

9. Posner A and Gorin G: Iridoschisis and glaucoma. Am J Ophthalmol 1958, 45: 451-2.

10. Albers EC and Klien BA: Iridoschisis. A clinical and histopathologic study.Am J Ophthalmol 1958, 46: 794-802.

11. Payne TD and Thomas RP: Iridoschisis. A case report. Am J Ophthalmol 1966, 62: 966-7.

12. Mills PV: Iridoschisis. Br J Ophthalmol 1967, 51: 158-64.

13. Duncan EN and Rieser JC: Iridoschisis. Arch Ophthalmol 1976, 94: 2004-5.

14. Zimmerman TJ, Dabazies OH Jr, Kaufman HE: Iridoschisis: a case report. Ann Ophthalmol 1981, 13: 297-8.

15. Weseley AC and Freeman WR: Iridoschisis and the corneal endothelium. Ann Ophthalmol 1983, 15: 955-64.

16. Romano A, Treister G, Barishak R, Stein R: Iridoschisis and angle-closure glaucoma. Ophthalmologica 1972, 164: 199-207.

17. Rodrigues MC, Spaeth GL, Krachmer JH, Laibson PR: Iridoschisis associated with glaucoma and bullous keratopathy. Am J Ophthalmol 1983, 95; 73-81.

18. Summers CG, Doughman DJ, Letson RD, Lufkin M: Juvenile iridoschisis and micropthalmos. Am J Ophthalmol 1985, 100: 437-9.
19. Pearson PA, Amrien JM, Baldwin LB, Smith TJ: Iridoschisis associated with syphilitic interstitial keratitis. Am JOphthalmol 1989, 107: 88-9.

20. Hyams S: Angle-closure Glaucoma. Amsterdam. Kugler and Ghedini Publications 1990, 39.

21. Lowe RF and Lim ASM: Primary Angle Closure Glaucoma. Singapore, PG Publishing 1989, pp 3, 35.

22. Anderson DR and Davis EB: Sensitivities of ocular tissues to acute pressure-induced ischemia. Arch Ophthalmol 1975, 93: 267-74.

23. Carnevalini A, Menchini U, Bandello F, Scialdone A, Brancato R: Aspects fluoroiridographiques de l'iridoschisis. $\mathrm{JFr}$ Ophtalmol 1988. 1: 329-32.

24. Winstanley J: Iris atrophy in primary glaucoma. Trans Ophthalmol Soc UK 1961, 81: 23-37.

25. Duke-Elder S and Perkins ES: Diseases of the Uveal Tract. In Duke Elder, S (ed) System of Ophthalmol, Vol 9. St. Louis, C.V. Mosby 1966, pp 694-8.

26. Cross HE and Maumenee AE: Progressive spontaneous dissolution of the iris. Surv Ophthalmol 1973, 18: 186-99.

27. Shields MB, Campbell DG, Simmons RJ: The essential iris atrophies. Am J Ophthalmol 1978, 85: 749-59.

28. Shields MB, Buckley E, Klintworth GK, Thresher R: Axenfeld-Rieger syndrome. A spectrum of developmental disorders. Surv Ophthalmol 1985, 29: 387-409. 